\title{
Echanges de phosphore entre les sédiments et l'eau. Influence de la dilution sur les cinétiques de traçage isotopique ${ }^{1}$
}

\author{
J. Capblancq2 \\ L. Labroue ${ }^{2}$ \\ J.C. Fardeau ${ }^{3}$
}

Mots clés : phosphore, échanges sédiment-eau, traçage isotopique.

Les cinétiques de traçage isotopique des échanges entre les ions phosphates en solution et les sédiments en suspension permettent de définir des indices caractérisant les potent ialités de transfert du P entre les sédiments et l'eau. L'utilisation de cette méthode nous a permis de déterminer une quantité de phosphore isotopiquement échangeable dans différents types de sédiments; cette valeur est fonction de la durée d'échange, fixée ici à 24 heures $\left(E_{24}\right)$.

La dilution du sédiment provoque le passage en solution d'une fraction croissante de ce pool d'ions échangeables selon une loi $Q=a \cdot E_{24} \cdot S^{b}$ qui permet de calculer la concentration (Q) de phosphore dans l'eau connaissant la masse(S) de sédiments en suspension. L'épuisement du P le plus mobile et l'allongement de la phase d'adsorption qui résultent de la dilution du sédiment entrainent une modification des cinétiques d'échange.

Sediment-water exchange of phosphorus. The influence of sediment dilution on radiotracer kinetics.

Keywords : phosphorus, sediment-water exchange, radiotracer kinetics.

The kinetics behavior of radiotracer ${ }^{32} \mathbf{P}$ during exchange reactions between soluble phosphate ions and suspended sediments provides information on parameters that characterize $P$ transport potentiality between the solid phase and the water. The method used enabled us to quantify the isotopically exchangeable phosphorus in different types of sediments during a 24 hours echange period $\left(\mathrm{E}_{24}\right)$. It is evident that sediment dilution involves the solubilization of exchangeable ion pool, with the process following the relationship $Q=a \cdot E_{24} . S^{b}$. This equation allows a calculation of the $P$ concentration in water $(Q)$ as a function of weight of suspended sediment $(S)$. As the result of sediment dilution both the release of the most labile $\mathrm{P}$ and the lengthening of the adsorption phase involve a modification of exchange kinetics.

\section{Int roduction}

Le transport du phosphore dans les eaux superficielles est étroitement associé à celui des matières en suspension. Ce transport s'effectue pour l'essentiel en périodes de crues avec des phases de dépôt et de remises en suspension (Verhoff \& al. 1982), au

1. Travail réalisé dans le cadre de l'ASP a Eau * du PIREN.CNRS. 2. Laboratoire d'Hydrobiologie, UA 695 CNRS, Université Paul Sabatier, 118 route de Narbonne, 31062 Toulouse Cedex, France. 3. D.B.S.R.A. Centre d'études nucléaires de Cadarache, B.P.1, 13115 Saint-Paul-Lez-Durance, France. cours desquelles les échanges qui s'établissent entre les différentes fractions du phosphore (dissous et particulaire, minéral et organique, etc), déterminent in fine les proportions de phosphore dissous ou lié aux sédiments qui parviennent aux lacs. Ces proportions, ainsi que la mobilité du $\mathbf{P}$ des sédiments, figurent parmi les caractéristiques importantes dont dépend la relation établie entre la production de phytoplanc. ton d'un lac et sa charge en $P$ total (OCDE 1982). Les végétaux utilisent, en effet, le phosphore sous forme de $\mathrm{PO}_{4}-3$ dissous; ce dernier est plus ou moins en équilibre avec le $P$ en phase solide, mais les mesures de $\mathbf{P}$ total plus faciles à réaliser que celles de ses constituants, ne permettent pas d'évaluer les quantités de $P$ biologiquement disponibles dans une eau. 
Les recherches entreprises dans ce domaine au cours de la dernière décennie s'inspirent de méthodes utilisées en agronomie pour évaluer la fertilité phosphorique des sols : séparation des composés du $P$ à l'aide de réactifs chimiques, qui désorbent, complexent ou dissolvent les ions phosphates liés aux sédiments (voir références in Bostrom \& al. 1982) ; mesure de la fraction de phosphore des sédiments utilisable par des cultures d'algues (Golterman \& al. 1969. Williams \& al. 1980, Young \& de Pinto 1982, Hanna \& Dauta 1983); évaluation de la fraction échangeable à l'aide de résines échangeuses d'ions (Huettl \& al. 1979) ou par échange isotopique avec du 32P (Li \& al. 1973).

La potentialité d'utilisation du phosphore par les plantes peut être exprimée par la mesure du flux d'échange entre le solide et la solution (qui nous semble plus proche de la notion d'intensité que la concentration de $P$ en solution) et de la capacité du solide à soutenir ce flux (notion de capacité ; White \& Beckett 1964). Les cinétiques de traçage isotopique permettent, en principe, de mesurer ces valeurs dans une suspension du sol sans avoir à extraire le P mobile (Fardeau 1981, Fardeau \& Jappe 1982).

L'objectif essentiel de ce travail est de tester les possiblités d'utilisation de cette approche pour caractériser les potentialités de transfert du $P$ entre les sédiments et l'eau en fonction de la nature des sédiments et de leur dilution.

\section{Bases théoriques de la méthode}

Dans un système en état stationnaire, la mesure du flux brut eau - sédiment permet d'estimer le flux opposé i.e. le flux que l'on obtiendrait initialement en maintenant nulle la concentration en phosphate dans l'eau. Cette mesure peut être effectuée par traçage isotopique, en ajoutant au système en équilibre une très faible quantité de ${ }^{32} \mathrm{P}$ sans entraineur et en suivant l'évolution de la radioactivité $(R)$ de la solution en fonction du temps. Cette évolution, ou cinétique de dilution isotopique, est théoriquement fonction du nombre de compartiments homogènes dans lequel se distribue le traceur.

On peut considérer le $\mathrm{PO}_{4}^{-3}$ en solution comme étant en équilibre avec les ions $\mathrm{PO}_{4}{ }^{-3}$ mobiles du solide répartis dans un nombre $q$ de sites d'échange possédant chacun ses propres caractéristiques cinétiques. La dilution isotopique dans le compartiment où est injecté le traceur est du type :

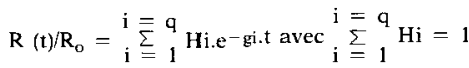

où $R_{t}$ représente au temps $t$ la radioactivité du compartiment marqué ; $R_{0}$ la radioactivité initiale $; i=$ $1,2, \ldots, q$ les indices des q compartiments; $\mathrm{H}$ et $\mathrm{g}$, des paramètres fonction de la taille des différents compartiments et des flux qui les relient.

On admet que les échanges entre les sites se font par auto-diffusion (Fardeau 1981 ; Barrow 1983). La similitude de (1) avec l'équation de diffusion en milieu fini (Crank 1975) permet de considérer les différents $\mathrm{Hi}_{i}$ comme les termes successifs d'une loi de répartition des différents $g_{i}$. On a alors :

$$
\mathrm{R}_{(\mathrm{t})} / \mathrm{R}_{\mathrm{o}}={ }_{\mathrm{i}=1}^{\mathrm{i}=\sum^{\mathrm{q}}} \mathrm{f}\left(\mathrm{g}_{\mathrm{i}}\right) \cdot \mathrm{e}^{- \text {gi.t }}
$$

où $\mathrm{g}_{\mathrm{i}}$ représente les constantes de vitesse d'échange des différents sites considérés chacun comme un compartiment ayant ses propres caractéristiques d'échange. Il est démontré (Fardeau 1981) que $\mathrm{f}\left(\mathrm{g}_{\mathrm{i}}\right)$ peut être exprimée par une fonction de distribution de probabilité "gamma ", dont la transformée de Laplace est une fonction puissance, numériquement très proche de :

$$
R_{(t)} / R_{o}=R_{1} / R_{o} \cdot t^{-n}
$$

où $\mathrm{R}_{\mathrm{t}}=$ radioactivité en solution au bout d'une minute, $\mathrm{n}=$ constante caractéristique de la cinétique.

Il est possible de définir, à partir de cette fonction, une constante moyenne d'échange $(\mathrm{gm})$ entre les ions en solution et l'ensemble des ions isotopiquement échangeables du solide :

$$
\mathrm{g}_{\mathrm{m}}=\mathrm{n} /\left(\frac{\mathrm{R}_{\mathrm{l}}}{\mathrm{R}_{\mathrm{o}}}\right) \frac{1}{\mathrm{n}} \text { (unités : } \mathrm{mn}-1 \text { ) }
$$

et un flux moyen d'échange $\left(\phi_{m}\right)$ entre ces deux compartiments

$$
\phi_{\mathrm{m}}=\mathrm{g}_{\mathrm{m}} \times \mathrm{Q} \quad \text { (unités : } \mu \mathrm{g} \text { P.mn }{ }^{-1} \text { ) }
$$

où $Q$ est la quantité d'ions phosphate en solution dans le système à l'état stationnaire. 
Cette cinétique permet enfin de mesurer le phosphore isotopiquement échangeable $\left(E_{t}\right)$, quantité qui varie avec le temps puisque :

$$
Q / R_{t}=E_{t} / R_{o}
$$

d'où en combinant (3) et (6) :

$$
\mathbf{E}_{\mathbf{t}}=\mathbf{Q} / \frac{\mathbf{R}_{\mathrm{I}}}{\mathbf{R}_{\mathrm{o}}} \cdot \mathbf{t}^{-\mathrm{n}}
$$

Les cinétiques de dilution isotopique permettent donc, en théorie, de décrire l'aptitude d'un sol ou d'un sédiment à libérer son phosphore par un paramètre d'intensité $\left(\phi_{\mathrm{m}}\right)$, un paramètre de quantité $\left(E_{t}\right)$ et un paramètre de capacité $\left(\phi \mathrm{m} / \mathrm{E}_{\mathrm{t})}\right.$.

\section{Matériel et méthodes}

Deux techniques complémentaires ont été utilisées pour définir les propriétés d'échange des sédiments en fonction de leur dilution.

Méthode A : dans $125 \mathrm{ml}$ de suspension aqueuse de sédiment, agitée une nuit pour obtenir un état stationnaire, on injecte à $t=0,1 \mathrm{ml}$ d'une solution de ${ }^{32} \mathrm{PO}_{4}$ sans entraîneur contenant une radioactivité $R_{0}$ comprise entre 0.05 et $0.5 \mathrm{MB}_{\mathrm{q}}$ selon la masse de sédiment mise en jeu. On prélève régulièrement dans ce mélange, à l'aide d'une seringue, $5 \mathrm{ml}$ de suspension que l'on filtre immédiatement sur des membranes de porosité $=0,2 \mu \mathrm{m}$. La radioactivité $R_{t}$ des solutions filtrées est mesurée par scintillation liquide. A l'issue du dernier prélèvement, on centrifuge le mélange pour doser la quantité $Q$ de phosphates en solution dans le surnageant et mesure la quantité $S$ de sédiment mis en ouvre.

Méthode $B$ : après un premier échange isotopique suffisamment long ( $t=12$ heures), le sédiment récupéré par centrifugation est remis immédiatement en suspension dans de l'eau non radioactive pendant 10 à 12 heures. Connaissant les quantités de radioélément en solution à l'issue du premier échange $\left(R_{t}\right)$ et de l'échange en retour $\left(R^{\prime}\right)$ et la quantité $Q^{\prime}$ de phosphates en solution à l'issue du deuxième échange, le rapport

$$
\mathbf{R}_{\mathrm{s}}^{\prime} / \mathbf{Q}^{\prime}=\mathbf{R}_{\text {sed }} / \mathbf{E}^{\prime} \mathrm{t}
$$

où $\mathbf{R}_{\text {sed }}=\mathbf{R}_{\mathrm{o}}-\mathbf{R}_{\mathrm{t}}=$ radioactivité des sédiments à la fin du $1^{\text {er }}$ échange, permet le calcul de la fraction échangeable et de sa répartition entre la solution et la phase solide.

La méthode de Stephens (1963) a été utilisée pour doser les ions phosphate en solution et le phosphore extrait des sédiments par minéralisation à chaud avec une solution de $\mathrm{H}_{2} \mathrm{SO}_{4}, 0,15 \mathrm{M}+\mathrm{K}_{2} \mathrm{~S}_{2} \mathrm{O}_{3}$.

Ces expériences ont été réalisées sur quatres sédiments d'origine différente:

- sédiments faiblement organiques recueillis dans la zone littorale de deux réservoirs, l'un riche en argile (C), l'autre plus limoneux (P.)

- sédiments très riches en matières organiques prélevés sur le fond d'un cours d'eau en aval d'un rejet urbain (M) et d'un canal dans une zone d'accu. mulation de feuilles mortes ( $R$ ).

\section{Résultats et discussion}

L'évolution, dans le temps, de la radioactivité restant en solution après un ajout de $32 \mathrm{P}$ à une suspension de sédiments $>2 \%$ en poids, et à l'état stationnaire, s'ajuste bien au modèle décrit par l'équation (3). Les paramètres de cette équation varient avec la nature des sédiments dont ils caractérisent le pouvoir fixateur vis-à-vis des ions $\mathrm{PO}_{4}-3$ (fig. 1).

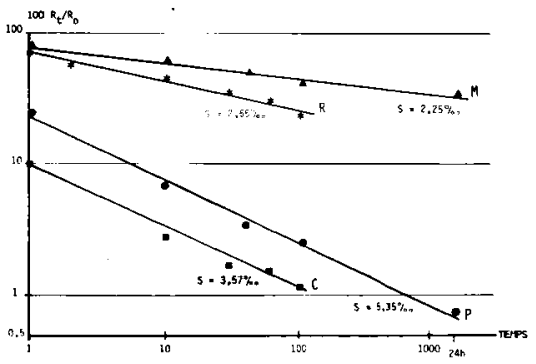

Fig. 1. Cinétiques de dilution isotopique d'ions ${ }^{32} \mathrm{PO}_{4}{ }^{-3}$ dans des suspensions sédiment-eau à l'etat stationnaire. 

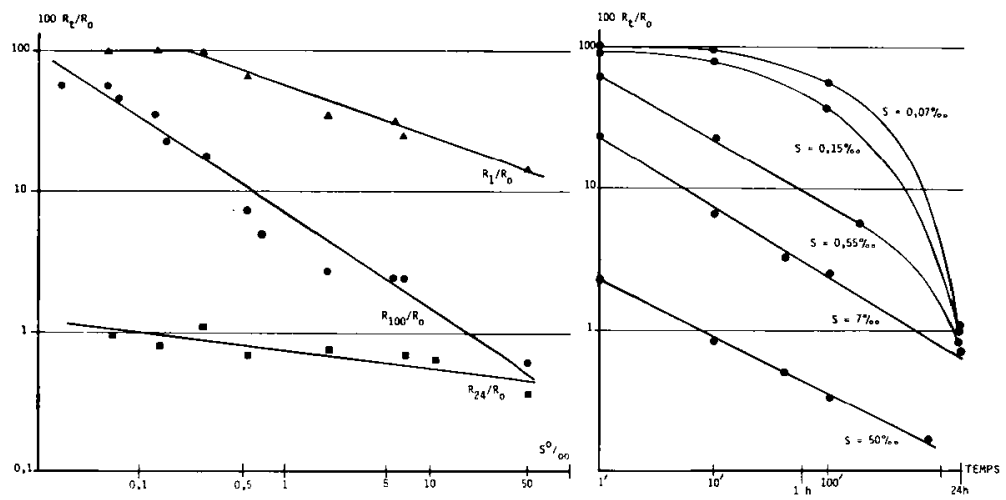

Fig. 2. Relations entre la dilution du sédiment $P\left(S \%\right.$ ( $\%$ rapport sédiment/eau en masse) et le pourcentage de ${ }^{32} \mathbf{P}$ en solution $\left(R_{t} / R_{0}\right)$ après une durée d'échange croissante $\left(R_{1}=1 \mathrm{mn} ; R_{100}=100 \mathrm{mn} ; R_{24}=24 \mathrm{~h}\right)$.

La valeur $R_{1} / R_{0}$ varie avec la dilution du sédiment i.e. avec la masse d'élément adsorbant. Pour des suspensions supérieures à $5-10 \%$, n peut être assimilé à une constante. Pour des valeurs inférieures à $1 \%$, cas général des sédiments en suspension dans les eaux naturelles, l'évolution est extrêmement rapide et la cinétique n'obéit plus au modèle $\mathrm{R}_{\mathrm{t}}=\mathrm{K} . \mathrm{t}^{-\mathrm{n}}$ (fig. 2).

On peut expliquer ce phénomène en considérant que la cinétique observée résulte d'une part des propriétés fixatrices du sédiment, d'autre part de la probabilité de rencontre des ions ${ }^{32} \mathrm{PO}_{4}$ avec les sites. Lorsque la masse de sédiments est suffisante, la diffusion est le facteur essentiel de diminution du $32 \mathrm{P}$ après 1 minute, d'où une faible variation du paramètre $\mathbf{n}$ qui représente bien les propriétés intrinsèques du sédiment. Par contre, une forte dilution diminue la probabilité de rencontre $32 \mathbf{P}$ - sédiments, ce qui a pour effet d'allonger la phase initiale d'adsorption et de masquer les propriétés diffusives du solide. La dilution, jouant par ailleurs le même rôle que l'appauvrissement du solide en phosphates (Barrow 1979), devrait augmenter le pouvoir fixateur du sédiment, i.e. le paramètre $n$. Les cinétiques observées sur 24 heures avec des dilutions croissantes s'éloignent alors de plus en plus de celle décrite par une fonction puissance de $\mathrm{t}(\mathrm{fig} .2)$.
Un échange isotopique de 24 heures (méthode A) et un échange aller-retour (methode B) pratiquées en parallèle sur un mème sédiment donnent des solutions de radioactivité spécifique $R_{24} / Q$ et $R_{\text {'s }}$ ! $Q$ ' sensiblement identiques (Tableau I). On peut donc considérer que le $\mathbf{P}$ échangeable qui participe à la désorption atteint pratiquement l'équilibre isotopique avec la solution pour des durées d'échange $<24$ heures. Cette conclusion est confirmée par le fait que, pour un sédiment et une radioactivité initiale donnés, la radioactivité spécifique des solutions reste à peu près constante, alors que les quantités de phosphore en solution ( $Q$ et $Q^{\prime}$ ) varient (Tableau I). Cependant, la légère décroissance de $R^{\prime} / Q^{\prime}$ observée lorsqu'on augmente la dilution des sédiments indique la mobilisation de sites moins marqués.

La quantité de $\mathbf{P}$ échangeable par unité de poids de solide est une constante, caractéristique du sédi. ment et indépendante du protocole expérimental (Tableau I). Sa détermination par voie directe ou indirecte (équation 7 ou 8) est cependant basée sur un dosage chimique des phosphates en solution et donc tributaire des techniques de séparation du solide et de la solution. Des essais de séparation par centrifugation et par filtration sur membranes de $0,45 \mu \mathrm{m}, 0,2 \mu \mathrm{m}$ et $0,1 \mu \mathrm{m}$ donnent des valeurs de 
Tableau I. Relations entre les quantités ${ }^{31} \mathbf{P}\left(Q\right.$ et $\left.Q^{\prime}\right)$ et de ${ }^{32} \mathrm{P}\left(\mathbf{R}_{24}\right.$ et $R$ 's) respectivement (1) en solution après un échange d'une durée de 24 heures après l'ajout de ${ }^{32} \mathrm{P}\left(\mathrm{R}_{\mathrm{o}}=8 \mu \mathrm{Ci} / 100 \mathrm{ml}\right)$ dans des suspensions de sédiment $\mathrm{P}, \mathrm{M}$ et $\mathrm{C}$ (en haut) et (2) libérées en 12 heures par ces mémes sédiments, remis en suspension et dilués, après marquage préalable par un échange d'une durée de 14 heures effectué dans les mémes conditions qu'en (1) (en bas). Les quantités de $P$ échan. geable $\left(E_{24}\right.$ et $E_{24}^{\prime}$ ) ont été calculées respectivement a l'aide des relations 6 et 8 (voir texte).

$\mathrm{S}_{00}=$ poids de sédiments en $\mathrm{g}_{1} \mathrm{1}^{-!} ; \mathrm{R}_{\mathrm{a} e \mathrm{u} !}=$ radioactivite des sédiments à l'issue du premier échange.

\begin{tabular}{|c|c|c|c|c|c|}
\hline Sédiment & ${ }^{5}$ & $\begin{array}{c}Q \\
n g+\ldots 1^{-1}\end{array}$ & $\begin{array}{c}R_{24} / Q \\
d p a . n g^{-1}\end{array}$ & $R_{0} / R_{24}$ & $\mathrm{Pg}^{\mathrm{E}_{24}}=1$ \\
\hline P & 20,9 & 25,0 & 51,77 & 152,4 & 349 \\
\hline M & 7,3 & 81,1 & 23,48 & 101.9 & 1132 \\
\hline c & 12,1 & 29,3 & 35,63 & 179,2 & 434 \\
\hline
\end{tabular}

\begin{tabular}{|c|c|c|c|c|c|c|c|c|c|c|c|c|}
\hline \multirow[b]{2}{*}{$5 \%$} & \multicolumn{4}{|c|}{$\mathbf{P}$} & \multicolumn{4}{|c|}{ H } & \multicolumn{4}{|c|}{ c } \\
\hline & 6,57 & 2,73 & 1,10 & 0,55 & 4,38 & 1,82 & 0,73 & 0,36 & 7,26 & 3,02 & 1,21 & 0,61 \\
\hline$Q^{\prime} \mathrm{ng} \cdot \mathrm{ml}^{-1}$ & 15,8 & 9,1 & 9.8 & 31,4 & 170,1 & 160.0 & 136.1 & 102,0 & 45,0 & 31,5 & 26,0 & 21,0 \\
\hline $\mathrm{R}_{1} / Q^{\prime} \mathrm{dpm} \cdot \mathrm{ng}^{-1}$ & 47.09 & 46,66 & 49.18 & 46,62 & 21.56 & 20,50 & 20,08 & 19.97 & 37,35 & 34,00 & 33.57 & 33,62 \\
\hline$R_{a \in d^{\prime}} / R_{g}$ & 151.5 & 111,0 & 38,5 & 6.35 & 30,4 & 14.2 & 6.79 & 4.54 & 66,6 & 43,4 & 21,3 & 13,2 \\
\hline$g_{24}+P^{2} \cdot g^{-1}$ & 364 & 366 & 343 & 362 & 1179 & 1239 & 1265 & 1286 & 413 & 453 & 457 & 469 \\
\hline
\end{tabular}

de $\mathrm{R}^{\prime}$ ( et donc de Q) sensiblement différentes. Ce problème était déjà évoqué par Mc Auliffe \& al, en 1947.

La dilution du sédiment se traduit par une aug-

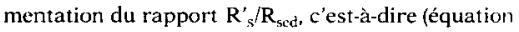
8) du rapport $P$ en solution $/ P$ isotopiquement échangeable. Les relations observées (fig. 3) peuvenı s'écrire :

$$
\mathrm{Q}=\mathrm{a} \cdot \mathrm{E}_{24} \cdot \mathrm{S}^{\mathrm{h}}
$$

où $\mathrm{Q}=$ phosphore en solution $\left(\mu \mathrm{g} \cdot \mathrm{I}^{-1}\right.$ de $\left.\mathrm{PO}_{4}-\mathrm{P}\right)$

$\mathrm{E}_{24}=$ phosphore isotopiquement échangeable en 24 heures ( $\left.\mu \mathrm{g} \cdot \mathrm{g}^{-1}\right)$

$\mathrm{S}=$ masse de sédiment par unité de masse d'eau ( $\mathrm{g}$ sédiments $\mathbf{1}^{-1}$ )

$\mathrm{a}$ et $\mathrm{b}=$ constantes caractéristiques du sédiment.

La quantité de $P$ en solution dépend donc directement de la teneur en $P$ échangeable du sédiment $\left(E_{24}\right)$ et, selon une fonction puissance, de sa dilution (Tableau II). Cette dilution épuise les compartiments les plus labiles du phosphore des sédiments au profit de la solution, ce qui doit donc augmenter le pouvoir fixateur du solide. Si l'on admet que la cinétique observée résulte d'une adsorption sur des particules à charge variable, suivie d'une diffusion dans le solide (Barrow 1983), le modèle graphique de la

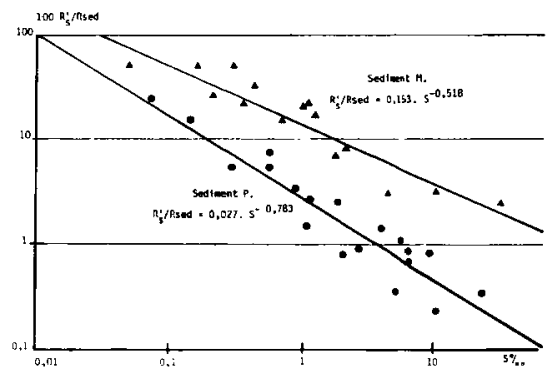

Fig. 3. Relations entre la dilution des sediments $P$ et $M$ et la proportion de $\mathbf{P}$ isotopiquement échangeable $\left(R^{\prime} / R_{\text {sed }}\right)$ en solution.

figure 4 explique les phénomènes observés. Un tel comportement correspond à l'observation selon laquelle les isothermes d'adsorption et de désorption du $\mathbf{P}$ ne présentent pas de phénomènes d'hystérésis si on considère le $\mathbf{P}$ échangeable (Kafkafi \& al 1967). Il explique également le fait que, si les quantités de $P$ total transportées par les rivières sont étroitement corrélées aux charges de matières en 
Tableau II. Indices caractéristiques des quatre sédiments étudiés. La quantité de phosphore en solution est exprimée à partir de la quantité de $\mathbf{P}$ échangeable $\left(E_{24}\right)$ et de la masse de sédiments en suspension ( $\mathbf{S}$ g. 1 l) par une équation déduite des relations représentées dans la figure 3. Les flux movens d'échange ( $\phi$ m) correspondent aux dilutions indiquées dans la colonne $\mathrm{S} \%$.

\begin{tabular}{|c|c|c|c|c|c|c|}
\hline Sédiment & $\begin{array}{l}P_{\text {total }} \\
\operatorname{mg} \cdot g^{-1}\end{array}$ & $\begin{array}{r}\mathrm{E}_{24} \\
\mathrm{mg} \cdot \mathrm{s}^{-1}\end{array}$ & $\mathrm{n}$ & $\begin{array}{c}Q=P \text { en solution } \\
\mu g \cdot 1^{-1}\end{array}$ & $\begin{array}{l}5 \\
\%\end{array}$ & $\oint_{m}$ \\
\hline $\mathbf{P}$ & 0,91 & 0,35 & $-0,484$ & $0,027 \cdot E_{24} \cdot s^{0,217}$ & 5,33 & 82 \\
\hline c & 2,86 & 0,47 & $-0,456$ & $0,0346 \cdot \mathrm{E}_{24} \cdot \mathrm{s}^{0,005}$ & 3,57 & 1271 \\
\hline$M$ & 2,66 & 1,17 & $-0,135$ & $0,153 \cdot E_{24} \cdot s^{0,482}$ & 2,24 & 202 \\
\hline $\mathbf{R}$ & 0,24 & 0,04 & $-0,205$ & $0,142 \cdot \mathrm{E}_{24} \cdot \mathrm{s}^{0,508}$ & 2,65 & 11 \\
\hline
\end{tabular}

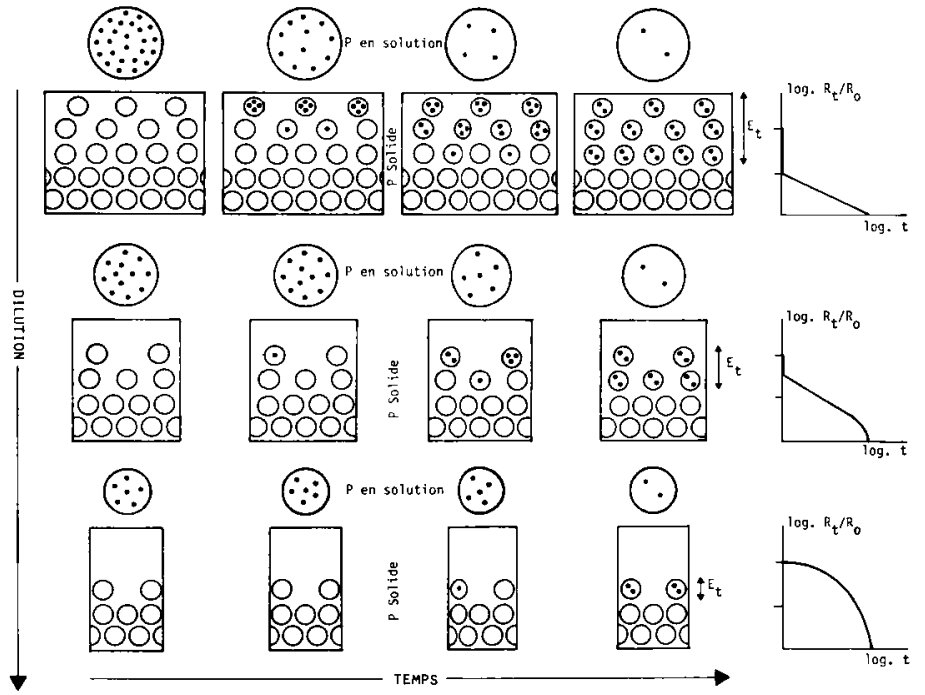

Fig. 4. Modèle conceptuel schématisant les relations entre le $\mathrm{P}$ en solution et le $\mathrm{P}$ des sédiments. Le ${ }^{32 P}$ (points noirs) introduit dans la solution à $t=0$ diffuse progressivement dans les sites d'échange du solide. La dilution du sédiment épuise les sites les plus facilement échangeables au profit de la solution et réduit en mênıe temps la surface d'adsorption. Les courbes d'évolution du ${ }^{32} \mathrm{P}$ en solution (a droite) résultent de la combinaison de ces processus. 
suspension et au débit, les teneurs en $\mathrm{PO}_{4}$ dissous varient de façon pratiquement indépendante (Golterman \& al. 1983). En effet, la proportion de $P$ en solution varie avec la masse de sédiments en suspension, ce qui a pour effet de réduire les fluctuations de concentration du $\mathbf{P}$ dissous dont la valeur maximum tend vers une limite fixée par le pouvoir fixateur du sédiment transporté.

Les cinétiques de traçage isotopique des échanges des ions phosphates entre l'eau et les sédiments en suspension permettent donc de définir une fraction de phosphore des sédiments susceptibles de subir un échange isotopique et dont il reste à démontrer qu'elle correspond à la fraction disponible pour les végétaux. Cette quantité de $P$ échangeable peut être calculée quelle que soit la dilution. Il n'en est pas de même pour la détermination des constantes moyennes d'échange $\left(\mathrm{g}_{\mathrm{m}}\right)$ et du flux d'échange $(\phi \mathrm{m})$ entre le solide et la solution, puisque la dilution fait varier les paramètres des cinétiques. La mesure des flux d'échange n'est possible que sur des suspensions de sédiments plus concentrées que les suspensions naturelles. Leur dilution, qui entraîne un épuisement du $\mathrm{P}$ labile analogue à celui qui résulterait d'une absorption par les végétaux, doit entrainer logiquement une diminution de $\mathrm{g}_{\mathrm{m}}$ et de $\phi_{\mathrm{m}}$ selon une fonction, qui reste à déterminer, de la fraction isotopiquement échangeable.

\section{Travaux cltés}

Barrow (N.J.) 1979. - The description of desorption of phosphate from soil. J. of Soil Science, $30: 259.270$.

Barrow (N.J.). 1983. - A mechanistic model for describing the sorption and desorption of phosphate by soil. $J$ of Soil Science, $34: 733-750$.

Bostrom (B.), Jansson (M.) \& Forsberg (C.), 1982. Phosphorus release from lake sediments. Ergebn. Limnol., $18: 5-59$.
Crank (J.) 1975. - The maihemarics of diffusion. 2d edition: Oxford University Press.

Fardeau (J.C.), 1981. - Cinétique de dilution isotopique et phosphore assimilable des sols. These Doctorat ès Sciences, Universite Paris VI, 198 p.

Fardeau (J.C.) \& Jappe (J.), 1982. - Intérêt des cinétiques d'échanges isotopiques pour la caractérisation du phosphore assimilable des sols. Science dis sol, 2: 113+124.

Golterman (H.L.), Bakels (C.C.) \& Jakobs-Moglin (J.) 1969. Availability of mud phosphates for the growth of algae. Verh. Intemat. Verein. Limnol., $17: 467-479$.

Gollerman (H.L.), Sly (P.G.) \& Thomas (R.L.), 1983. - Study of the relationship between water quality and sediment transport. Technical Papers in Hydrology; Unesco: $231 \mathrm{p}$.

Hanna (M.) \& Dauta (A.) 1983. - Bioassays : a comparative study of three parameters related to phosphorus bioavailability vield, growth rate and intracellular concentration of phosphorus). Ammls. Limnol, 19: 59-66.

Huettl (P.J.), Wendt (R.C.) \& Corey (R.B.), 1979. - Prediction of algal available phosphorus in runoff suspensions. J. Environ. Qual., $8: 130-132$.

Kafkafi (U.), Posner (A.M.) \& Quirk (J.P.). 1967. - Desorption of phosphate from kaolinite. Soil Science Soc. Amer. Proc., 31 : 348-353.

Li (W.C.), Armstrong (D.E.) \& Harris (R.F.), 1973. - Measurement of exchangeable inorganic phosphate in lake sediments. Environmenial Science and Technology, $7: 454456$.

McAuliffe (C.D.), Hall (N.S.), Dean (L.A.) \& Hendricks (S.B.), 1947. - Exchange reactions between phosphates and soils: hydroxylic surfaces of soil minerails. Soil Science Soc. Amer. Proc., 11: 119-129.

O.C.D.E. 1982. - Eutrophisation des eaux. Methodes de surveillance, d'évaluation et de lutte. O.C.D.E. Paris : 164 p.

Stephens (K.). 1963. - Détermination of low phosphate concentrations in lake and marine waters. Limmol. Oceanogr., 8 : 361.362 .

Verhoff (F.H.), Melfi (D.A.) \& Yaksich (S.M.), 1982. - An analysis of total phosphorts transport in river systems. Hydrobiologia, 91: 241-252.

White (R.E.) \& Beckett (P.H.T.). 1964. - Studies on the phosphate potentials of soils. 1 . The measurement of phosphate potential. Plant and Soil, 2: 1-16.

Williams (J.D.H.). Shear (H.) \& Thomas (R.L.). 1980. - Availability to Scenedesmus quadricauda of different forms of phosphorus in sedimentary materials in the Great Lakes. Limnol. Oceanogr., $25: 1.11$.

Young (T.C.) \& De Pinto (J.V.), 1982. - Algal-availability of parti. culate phosphorus from diffuse and point sources in the lower Great Lakes basin. Hydrobiologia, 91 : 111.119. 